\title{
3D global and regional patterns of human fetal subplate growth determined in utero
}

\author{
J. Corbett-Detig $\cdot$ P. A. Habas $\cdot$ J. A. Scott $\cdot$ \\ K. Kim • V. Rajagopalan • P. S. McQuillen • \\ A. J. Barkovich · O. A. Glenn · C. Studholme
}

Received: 28 May 2010/Accepted: 6 October 2010/Published online: 29 October 2010

(C) The Author(s) 2010. This article is published with open access at Springerlink.com

\begin{abstract}
The waiting period of subplate evolution is a critical phase for the proper formation of neural connections in the brain. During this time, which corresponds to 15 to 24 postconceptual weeks (PCW) in the human fetus, thalamocortical and cortico-cortical afferents wait in and are in part guided by molecules embedded in the extracellular matrix of the subplate. Recent advances in fetal MRI techniques now allow us to study the developing brain anatomy in $3 \mathrm{D}$ from in utero imaging. We describe a reliable segmentation protocol to delineate the boundaries of the subplate from T2-W MRI. The reliability of the protocol was evaluated in terms of intra-rater reproducibility on a subset of the subjects. We also present the first $3 \mathrm{D}$ quantitative analyses of temporal changes in subplate volume, thickness, and contrast from 18 to $24 \mathrm{PCW}$. Our analysis shows that firstly, global subplate volume increases in proportion with the supratentorial volume; the
\end{abstract}

J. Corbett-Detig - P. A. Habas - J. A. Scott - K. Kim .

V. Rajagopalan · C. Studholme

Biomedical Image Computing Group, Department of Radiology and Biomedical Imaging, University of California San Francisco,

San Francisco, CA, USA

e-mail: james.corbett-detig@uvm.edu

J. Corbett-Detig - P. A. Habas - J. A. Scott - K. Kim ·

V. Rajagopalan - A. J. Barkovich · O. A. Glenn · C. Studholme Department of Radiology and Biomedical Imaging, University of California San Francisco, San Francisco, CA, USA

P. S. McQuillen

Department of Pediatrics, University of California San

Francisco, San Francisco, CA, USA

C. Studholme $(\square)$

University of California San Francisco, Box 0628, Slot 6,

San Francisco, CA 94143-0628, USA

e-mail: colin.studholme@ucsf.edu subplate remained approximately one-third of supratentorial volume. Secondly, we found both global and regional growth in subplate thickness and a linear increase in the median and maximum subplate thickness through the waiting period. Furthermore, we found that posterior regions-specifically the occipital pole, ventral occipitotemporal region, and planum temporale —of the developing brain underwent the most statistically significant increases in subplate thickness. During this period, the thickest region was the developing somatosensory/motor cortex. The subplate growth patterns reported here may be used as a baseline for comparison to abnormal fetal brain development.

Keywords Fetal imaging - Tissue segmentation . Anatomical MRI - Subplate - Brain development . 3D thickness and volume

\section{Introduction}

In the course of the human brain development, growth of cerebral mantle is readily visualized by magnetic resonance imaging (MRI). Specifically, several transient zones including the germinal matrix (ventricular and subventricular zones), subplate (SP), intermediate zone (IZ), and cortical plate $(\mathrm{CP})$ are visible during development of the fetal brain (Kostovic et al. 2002). Like other fetal zones of the cerebral mantle, the SP is a heterogeneous structure with a variety of neurons, local and projection circuits, and extensive extracellular matrix (ECM) (for review, see McQuillen and Ferriero 2005 or Chan et al. 2002). The SP is also the site of critical developmental processes in the fetal brain. The SP forms transient circuits with thalamocortical and cortico-cortical axons pausing in their axonal projection to the CP (Rakic 1976; Kostovic and Rakic 
1990). This development of transient networks is known as the waiting period and was first described in fetal rhesus monkey visual cortex (Rakic 1976) and later in humans (Kostovic and Goldman-Rakic 1983). During the waiting period, the SP expands to varying degrees across the brain (Kostovic and Rakic 1990; Perkins et al. 2008).

The time course of the SP development in the human is described in organizational stages by Kostovic and Rakic (1990) as follows: presubplate stage (10-12 postconceptual weeks, PCW), subplate formation stage (13-15 PCW), subplate stage (15-35 PCW), and subsequent subplate dissolution stage (post $35 \mathrm{PCW}$ ) (Kostovic and Rakic 1990). During the subplate stage, which encompasses the waiting period, the SP develops into the most prominent zone of the developing telencephalon and reaches a thickness that is up to four or five times greater than that of the CP (Kostovic and Rakic 1990; Kostovic and JovanovMilosević 2006; Perkins et al. 2008). Regional differences in the timing and quantity of thalamic and cortical afferents to subplate are associated with the thickness. For example, thalamocortical innervation of the SP deep to the somatosensory cortex occurs prior to thalamocortical innervation of the SP deep to the primary visual cortex and is multiple times thicker (Kostović and Judas 2010).

Previous work quantifying measurements of the SP has, for the most part, focused on post-mortem histology and more recently 2D T1-weighted (T1-W) and T2-weighted (T2-W) MR images (Kostovic and Rakic 1990; Kostovic et al. 2002; Perkins et al. 2008; Widjaja et al. 2010). Histological studies have reported thickness measurements with the aid of various cellular stains. SP thickness has previously been reported from 2D distance measures up to $5 \mathrm{~mm}$ (Kostovic and Rakic 1990). These measurements are by no means uniform. In the visual cortex, the SP is very thin around the calcarine fissure yet two to three times thicker in the regions superior and inferior to the calcarine fissure (Kostovic and Rakic 1990). While an effective 2D tool, the classic histological approach cannot measure SP thickness out of the plane of section and therefore, may not be the most effective method to study the SP in more complex and curved areas of the cerebral mantle.

Improvements in MR image acquisition have made in utero $2 \mathrm{D}$ measurements possible. The overlap between histological data and MR images was previously verified; it was found that changes in the signal of T1-W MR images accurately reflected histological changes, in a large part, indirectly reflecting the ECM content (Kostovic et al. 2002). A recent study that used point estimates also confirmed that 2D T2-W MR images accurately reflect histological changes for the human SP (Perkins et al. 2008). This study showed that the SP could be up to $4.5 \mathrm{~mm}$ thick on 2D T2-W MR images. To date, no study has mapped the thickness of SP across the entire hemispheric extent or calculated average patterns of thickness for a range of subjects to examine its pattern of growth and how this pattern changes with increasing gestational age.

Recent developments in motion correction and 3D reconstruction (Rousseau et al. 2006; Kim et al. 2010) provide the ability to measure $3 \mathrm{D}$ volume and thickness of tissue regions in utero. In this study, we use these tools to analyze 3D MR images of the human fetal brain acquired in utero to characterize SP growth during the waiting period. Specifically, our study aims to: (1) develop a reliable protocol to manually delineate the developing SP on motion-corrected 3D clinical MRI data and (2) analyze SP volume and thickness in 3D for the period of development which corresponds to part of the waiting period visible in these imaging studies. These findings will provide the first maps of local thickness and volume measures of normal subplate growth in utero during this key neurodevelopmental stage. We hypothesize that changes in the volume of the SP will be correlated with parallel changes in the supratentorial brain volume (STV). Furthermore, we hypothesize that SP thickness will be spatially varying and the rate of growth will not be uniform across the brain, which may be in part due to the differences in the amount and the timing of arriving afferents.

\section{Materials and methods}

\section{Subjects}

Twenty-one subjects were selected from the age range 20.57 to 25.86 gestational weeks (GW) calculated from last menstrual period (mean: $23.14 \mathrm{GW}$, standard deviation: $\pm 1.59 \mathrm{GW})$. Subjects were selected from a pool of control subjects as part of a larger fetal MRI study. The study was approved by the Institutional Review Board and written informed consent was obtained. Control subjects were either volunteers recruited by informational flyers on the medical center campus or were referred for a clinical scan. None of the fetuses or their mothers were sedated during the MR examination. All MR scans were reviewed by a pediatric neuroradiologist (O.A.G.) who determined that each fetal brain was normal in appearance. All subjects were born healthy at term.

\section{MR acquisition}

MR images were collected on a $1.5 \mathrm{~T}$ magnet (GE Healthcare, Milwaukee, WI) using an eight-channel torso phased-array coil. Multiple stacks of single-shot fast spinecho (SSFSE) T2-W images were acquired in approximately planned axial, sagittal, and coronal planes without gap. The acquired voxel dimensions were approximately 
$0.5 \times 0.5 \times 3 \mathrm{~mm}$. Because of clinical protocol the TR $(6,400 \pm 2,600 \mathrm{~ms})$ and TE $(90.6 \pm 0.6 \mathrm{~ms})$ varied. Each stack contained at least 20 slices. On average, between two and three stacks per orientation were used for each reconstruction, depending on data acquired data quality, and the absence of in-slice fetal motion. To adjust for between slice fetal motion, the individual slices of each subject were collectively co-registered with the slice intersection motion correction (SIMC) technique (Kim et al. 2010). Then the registered images were reconstructed to create a single $3 \mathrm{D}$ volume image in approximately axial orientation with an isotropic voxel dimensions of the acquired in-plane pixel size of around $0.5 \mathrm{~mm}$ (Kim et al. 2010). The reconstructed 3D images were then manually segmented and analyzed in their 3D reconstructed orientation.

\section{Volumetric analyses}

Previous manual segmentations of STV (telencephalon and diencephalon) were divided into $\mathrm{CP}$, white matter (WM), germinal matrix, and the lateral ventricles (Habas et al. 2010a; Habas et al. 2010b). Calculation of STV was the sum of the CP, WM, and germinal matrix tissue label volumes. Specifically, the WM label encompassed IZ, SP, and deep gray nuclei. Since in utero MRI does not have the resolution or contrast to visualize two cell dense regions only a few millimeters thick, we defined the germinal matrix as all proliferative zones (ventricular and subventricular zones) surrounding the ventricles. These tissue labels were used as a foundation for the segmentation of the SP. The SP segmentations were completed in the coronal plane and confirmed in 3D from the axial and sagittal planes. The SP was manually segmented with the RView image display tool (http://rview.colin-studholme. net/) running under UBUNTU linux.

The protocol for tracing the SP was developed by comparing the reconstructed isotropic images with published histological and MR data (Bayer and Altman 2005; Kostovic and Rakic 1990; Kostovic et al. 2002; Larroche and FeessHiggins 1987; Perkins et al. 2008; Rados et al. 2006). The general MR T2-W appearance of laminar tissue showed several structures of alternating intensities (Glenn 2009 and Bystron et al. 2008): the germinal matrix (hypointense), the IZ (hypo and hyperintense bands), the SP (hyperintense), and the CP (hypointense) and therefore, the SP appeared hyperintense compared to surrounding tissues. Specifically, the SP has two major boundaries: (1) a superficial border with the $\mathrm{CP}$ and (2) a deep border with the IZ. The SP is subjacent to boundary-1, which was already delineated (Habas et al. 2010a, b) and confirmed in our tracing, whereas boundary-2 was defined and traced in this study.

Boundary-2, the deeper border, was defined primarily in the coronal plane using local intensity differences between the SP and adjacent tissues. Boundary-2 was defined in relation to the ventricles and extending concentric layers of the IZ. In the frontal lobe, boundary-2 encircled the IZ (Fig. 1b). Boundary-2 intersected boundary-1 at the midline of each hemisphere suprajacent to the corpus callosum and this was used as the starting point for boundary-2 delineation (Fig. 1c). In general, there were two visible hypointense bands in the IZ in the frontal lobe (Fig. 1f). The outer band formed the deep boundary (boundary-2) of the SP. In the presence of only one visible band, the single band was used to form boundary-2. Moving in the posterior direction, the IZ became very thin near the insular cortical region and the deep gray nuclei extended more lateral. This invoked a change in segmentation procedure. The most lateral portions of the deep gray nuclei, which were hypointense, served as the deep border with the SP (Fig. 1d). Between the deep gray nuclei and SP lies external capsule and claustrum, which is not clearly distinguishable in the images. Therefore, SP in the insular region may be slightly over represented. Superior and inferior to the deep gray nuclei, the outer-most hypointense band of the IZ persisted as the boundary with SP. Furthermore, the SP tapered at the intersection of boundary- 1 and boundary- 2 in the temporal lobe. Continuing in the posterior direction on the coronal plane, the lateral portions of the IZ returned and replaced the deep gray nuclei as a border with SP (Fig. 1e). This coincided with the appearance of the temporal horns. In the occipital region, the hyperintense and hypointense bands of the IZ were less distinct than anterior regions. Therefore, the outer-most continuous hypointense band was applied as the SP border of boundary-2. As in the frontal pole, the SP in the occipital pole was a continuous layer encircling the IZ. As a general rule, SP continuity was maintained as much as possible.

3D volume and mean intensity, and laminar thickness analyses

Global tissue volumes were calculated in RView for each tissue label-the CP, IZ, SP, the germinal matrix, and the lateral ventricles. We performed linear regression with statistical significance to analyze the relationship between volume and gestational age. In addition, mean intensities of the aforementioned zones were abstracted from the segmentation. The mean intensities of the SP and IZ were used to establish an intensity contrast ratio of boundary-2, which was then analyzed by linear regression with age.

Calculation of laminar thickness and statistical analyses of regional changes in SP thickness

The thickness of the SP and CP was estimated using a previously developed voxel-based method for cortical 
Fig. 1 Example motioncorrected and 3D reconstructed fetal brain images used in this study. The subplate (SP) is a transient fetal structure located between the white and black dotted lines. The white dotted line corresponds to the boundary between SP and the $\mathrm{CP}$ whereas the black dotted line corresponds to a second boundary between the SP and intermediate zone. The SP was first segmented in the coronal view and verified in the axial and sagittal planes. a Axial slice of reconstructed image shows the position of $\mathbf{b}-\mathbf{e}$ which progress in the posterior direction. b Slice of the developing frontal lobe which shows the developing SP encircling the intermediate zone. $\mathbf{c}$ The emergence of the corpus callosum (marked by an arrow). d Lateral portions of the basal ganglia (marked by arrows). e Occipito-parietal view of the developing fetus showing the tapered appearance of the developing SP. f Sagittal view of the developing SP showing laminar tissue layers
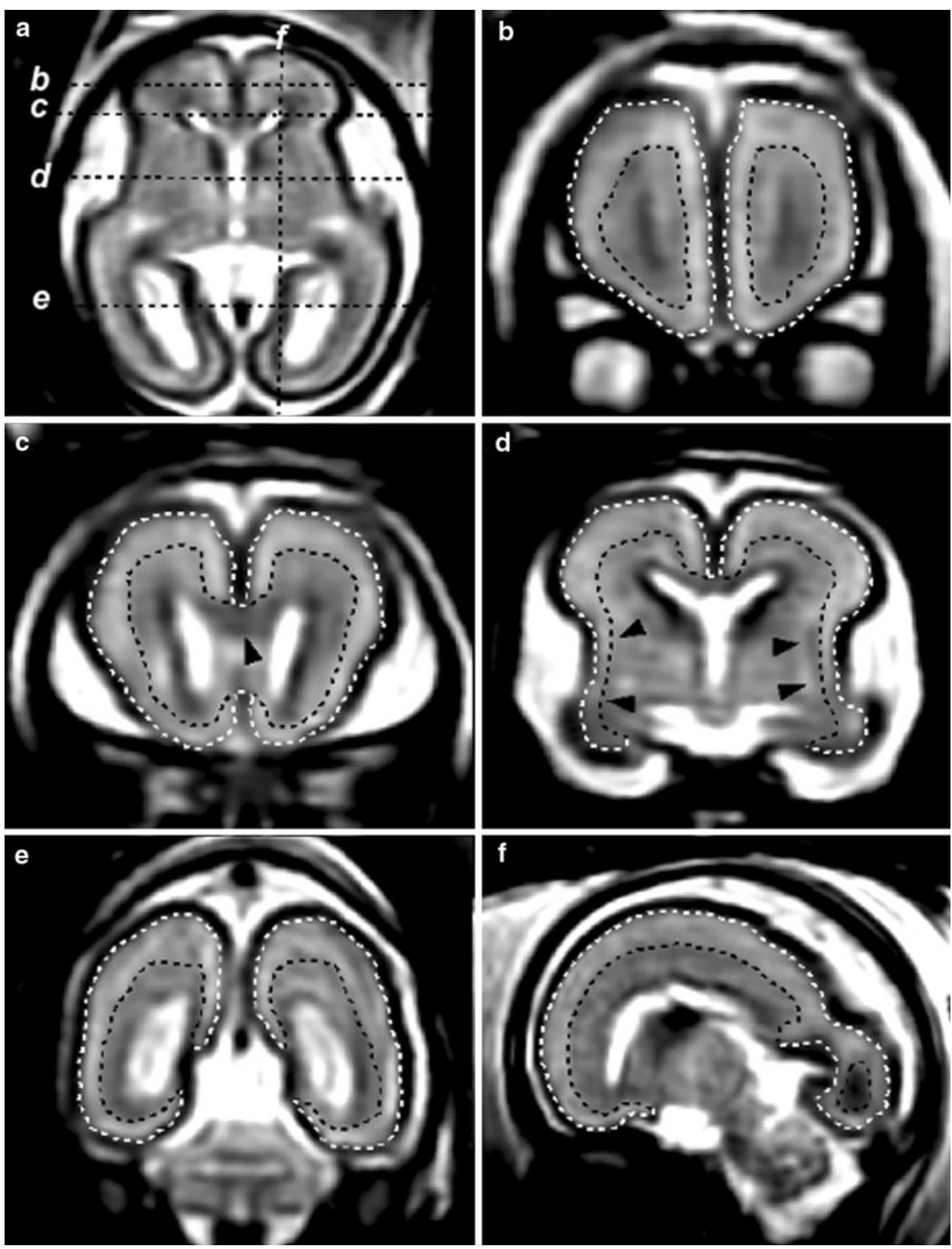

thickness (Jones et al. 2000; Yezzi and Prince 2003). This method defines non-intersecting paths between points on the inner and outer surface of each tissue label. The length of each path was used as unique thickness measurements for SP and CP and both were mapped to points on the outer SP surface (boundary-1). The SP and CP thickness maps were smoothed with a Gaussian kernel to minimize local variation and allow for the examination of consistent patterns on the tessellated surface corresponding to boundary-1.

To analyze the common patterns of thickness and its change across the study population, a group-wise non-linear registration was performed to form an average fetal brain shape (Habas et al. 2010a). These non-linear transformations were used to warp individual thickness and thickness ratio maps to a common coordinate frame of the average fetal anatomy and then to a tessellated surface corresponding to boundary-1. To exclude surface points for which no meaningful thickness measure existed in a given subject, a binary mask was created based on the logical combination of regions in each subject where the thickness estimate was zero. The pattern of regional change was statistically examined through the age range of our study using a linear regression of these scalar thickness values computed at every vertex along the average surface. $t$ tests were performed to evaluate the significance of the age effect over the whole surface and significance was then corrected for multiple 
comparisons using permutation testing (Nichols and Holmes 2002).

\section{Results}

Intra-rater reliability of SP segmentation was determined by Dice similarity coefficients (DSC). The DSC value of the right hemispheres of four subjects traced twice ranged from 0.86 to 0.91 indicating very good agreement and confirmed the reproducibility of the tracing protocol.

The contrast ratio of the mean intensities of the SP and IZ decreased with age due to convergence of both zones' mean intensities. The ratio of mean SP intensity to mean IZ intensity decreased linearly with age $\left(R^{2}=0.75\right.$, $p<0.001)$ from 1.09 to $1.02(1.06 \pm 0.02)$. At $26 \mathrm{GW}$, the ratio approached a numerical value of 1 , which indicated no contrast between the mean intensities of the SP and IZ (Fig. 2).

SP volume across all subjects ranged from $9.89 \mathrm{~cm}^{3}$ (20.57 GW) to $29.02 \mathrm{~cm}^{3}(25.57 \mathrm{GW})\left(18.95 \pm 3.23 \mathrm{~cm}^{3}\right)$. The volume of the SP increased linearly with age at a rate of $3.32 \mathrm{~cm}^{3}$ per GW $\left(R^{2}=0.92, p<0.001\right)$ (Fig. 3a). During the same period the STV is best modeled as linear growth $\left(R^{2}=0.94, p<0.001\right)$. The ratio of SP volume to STV does not significantly change with age $(33.03 \pm 1.83 \%)$ (Fig. 3b).

Average age-independent surface maps generated from all subjects represent the general spatial pattern of SP thickness (Fig. 4). On the average surface, the SP thickness varied between 0.5 and $4.9 \mathrm{~mm}(3.0 \pm 1.1 \mathrm{~mm})$ and $\mathrm{CP}$ thickness values varied from 0.86 to $2.6 \mathrm{~mm}$ $(2.17 \pm 0.26 \mathrm{~mm})$. The average $\mathrm{CP}$ thickness did not vary

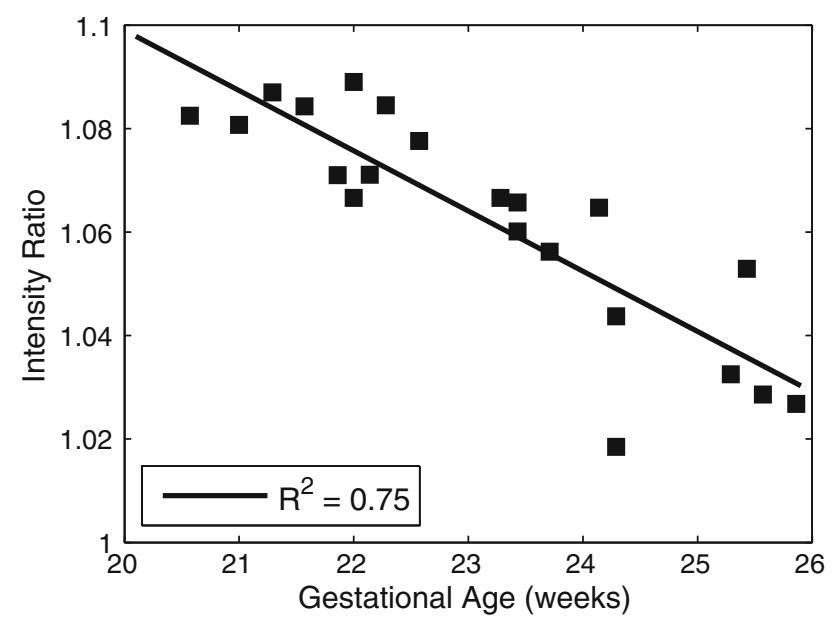

Fig. 2 Measurements of decreasing T2-W MRI contrast between the developing SP and intermediate zone, as shown by ratio of the mean intensities of the SP and the intermediate zone from 20 to 26 gestational weeks by last menstrual period (GW)
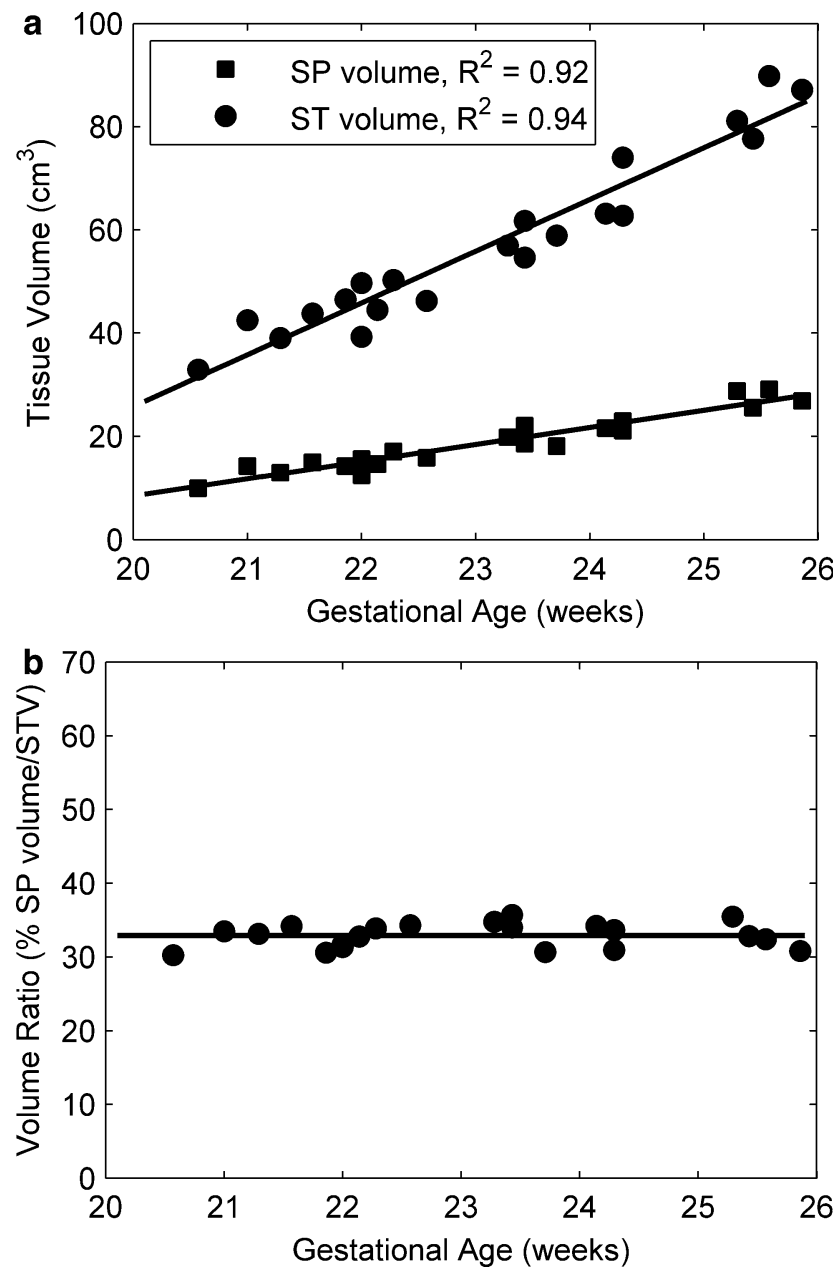

Fig. 3 Estimates of volumetric growth in the SP and supratentorial volume (STV) during the waiting period. a Linear growth of the SP and ST absolute volumes. b The volumetric ratio of the SP to the STV does not change between 20 and $26 \mathrm{GW}$

as much as that of SP. The ratio of SP to CP thickness varied from less than 1 to $2.12(1.38 \pm 4.76)$. Examination of the spatial pattern revealed that the SP was thickest in the developing superior part of the temporal and the frontoparietal regions. These general characteristics of the spatial pattern were found in all individual subjects' thickness maps.

The SP thickness also showed regional variation in growth. Initially, the thickest regions were concentrated in fronto-parietal region. Over time, SP thickness increased in the regions anterior and posterior to the fronto-parietal region (Fig. 5). Specifically the occipito-parietal region, frontal lobe, and the presumptive planum temporale showed the greatest increase in the SP thickness. The SP median thickness increased linearly with age (Fig. 6) $\left(R^{2}=0.80, p<0.001\right)$, as did the SP maximum thickness (Fig. 6) $\left(R^{2}=0.73, p<0.001\right)$. Individual values of the median cortical thickness varied from $1.92 \mathrm{~mm}$ 


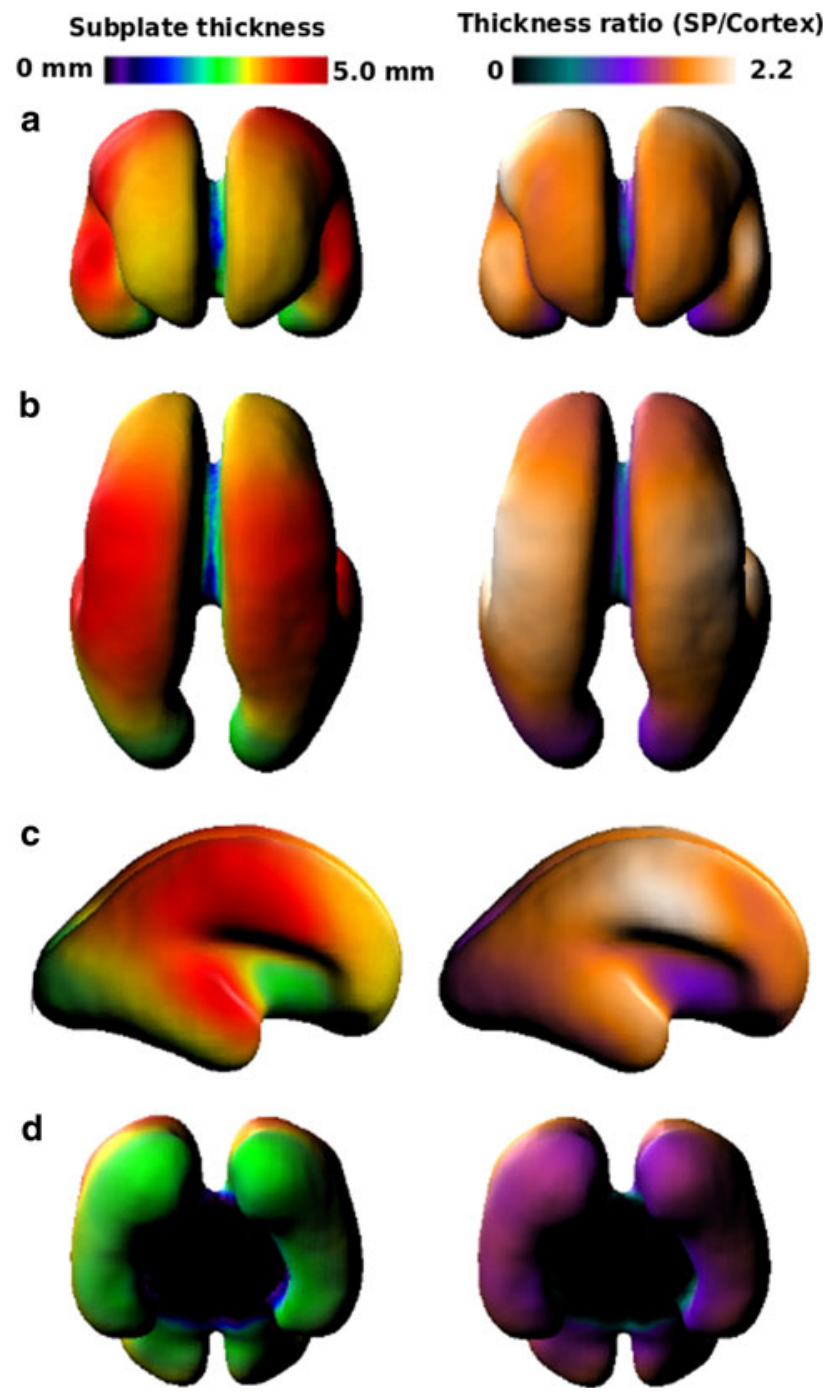

Fig. 4 Summary of views of the average SP thickness map and the average thickness ratio (SP thickness/CP thickness) map projected on to an average surface mesh of the SP. The ratio map mirrors the SP map in part due to the uniform thickness of the CP. a Anterior view of the developing frontal lobe. b Superior view of the developing occipital, parietal, and frontal lobes. c Lateral view of the SP. d Inferior view of the ventral occipital lobe

$(20.57 \mathrm{GW})$ to $2.84 \mathrm{~mm}(25.86 \mathrm{GW})(2.19 \mathrm{~mm} \pm 0.28$ $\mathrm{mm})$. Furthermore, the median ratio varied from 1.31 $(24.29 \mathrm{GW})$ to $1.84(25.43 \mathrm{GW})(1.53 \pm 1.13)$ and the maximum ratio varied from $2.17(25.57 \mathrm{GW})$ to 3.40 $(25.43 \mathrm{GW})(2.59 \pm 0.27)$.

At approximately $30 \%$ of the points on the average surface, SP thickness increased with age $(p<0.01)$. Specifically, areas of significant increases in SP thickness included the superior regions of the temporal lobe (Fig. 7a, b), the lateral portions of the occipital lobe (Fig. 7c), and the ventral surface of the developing occipital and temporal lobes (Fig. 7d). Aside from an area of particularly rapid growth on presumptive planum temporale (reddish regions of Fig. 7a, b), the rates of thickness growth associated with these regions were similar to each other.

\section{Discussion}

In this study, we developed a protocol for the 3D delineation of the SP from motion corrected in utero MRI, based upon T2-W tissue intensity properties. The reproducibility of the procedure was evaluated by intra-rater reliablity on a subset of images. From these segmentations, we were able to extract volumetric data and thickness maps for SP and $\mathrm{CP}$. We have estimated the growth rate of the SP and shown that the ratio of SP volume to STV is stable from 20 to $26 \mathrm{GW}$ (approximately 18 to $24 \mathrm{PCW}$ ). The thickness maps revealed a distinct spatial pattern that is consistent with previous slice-based estimates of SP thickness from histological and 2D MR analyses (Girard and Raybaud 1992; Kostovic and Rakic 1990; Kostovic et al. 2002; Perkins et al. 2008; Widjaja et al. 2010). Furthermore, we located regions of locally greater SP expansion with age from 3D surface-based thickness maps. This observed growth pattern was likely due to locally varying rates of neuropil and ECM increases and the addition of afferent fibers (Kostovic and Rakic 1990). Another source of growth in the second trimester may be associated with the arrival late-born SP cells (Hevner and Zecevic 2006).

Prior to $20 \mathrm{PCW}$, growth of the SP is quite rapid and non-linear, and then subsequently slows during the waiting period reaching maximum thickness between 29 and 31 PCW (Kostovic et al. 2002; Kostovic and Rakic 1990; Samuelsen et al. 2003). Our study, showed steady, linear increases in SP volume, global median and local maximum SP thickness, which suggests continued SP growth past $26 \mathrm{GW}$ (24 PCW) even though the SP could not be distinguished from IZ on the T2-W images. Previous studies showed that thickness is associated with the amount of cortico-cortical input to a region and is negatively correlated with cell density (Kostovic and Rakic 1990; Kostovic et al. 2002). Consistent with previous reports, we found that SP subjacent to developing precentral and postcentral gryal areas had the greatest thickness and the thinnest regions were in the ventral and medial occipital lobes (Kostovic and Rakic 1990). As expected from the different time courses for thalamocortical and cortico-cortical afferent arrival to the SP, we saw a symmetrical and regionally specific pattern of SP growth from 20 to $26 \mathrm{GW}$. For the age range of our study, the thickness of the SP increased in a regional manner that agrees with previous reports (Kostovic and Rakic 1990; Perkins et al. 2008; for review, Kostovic and Jovanov-Milosević 2006). For example, much of the occipital SP is growing at a rate of $30 \%$ per $\mathrm{GW}$, yet there is not significant change in 
Fig. 5 Example SP thickness maps from individual subjects across the age range of our study. The SP is initially thickest in the fronto-parietal region. Over the range of this study the SP thickens in regions anterior and posterior to the fronto-parietal region. Note that the SP does not connect as shown in the figure, but that is an artifact of generating the average fetal SP. We accounted for this in our statistical analysis by applying a mask to exclude such regions

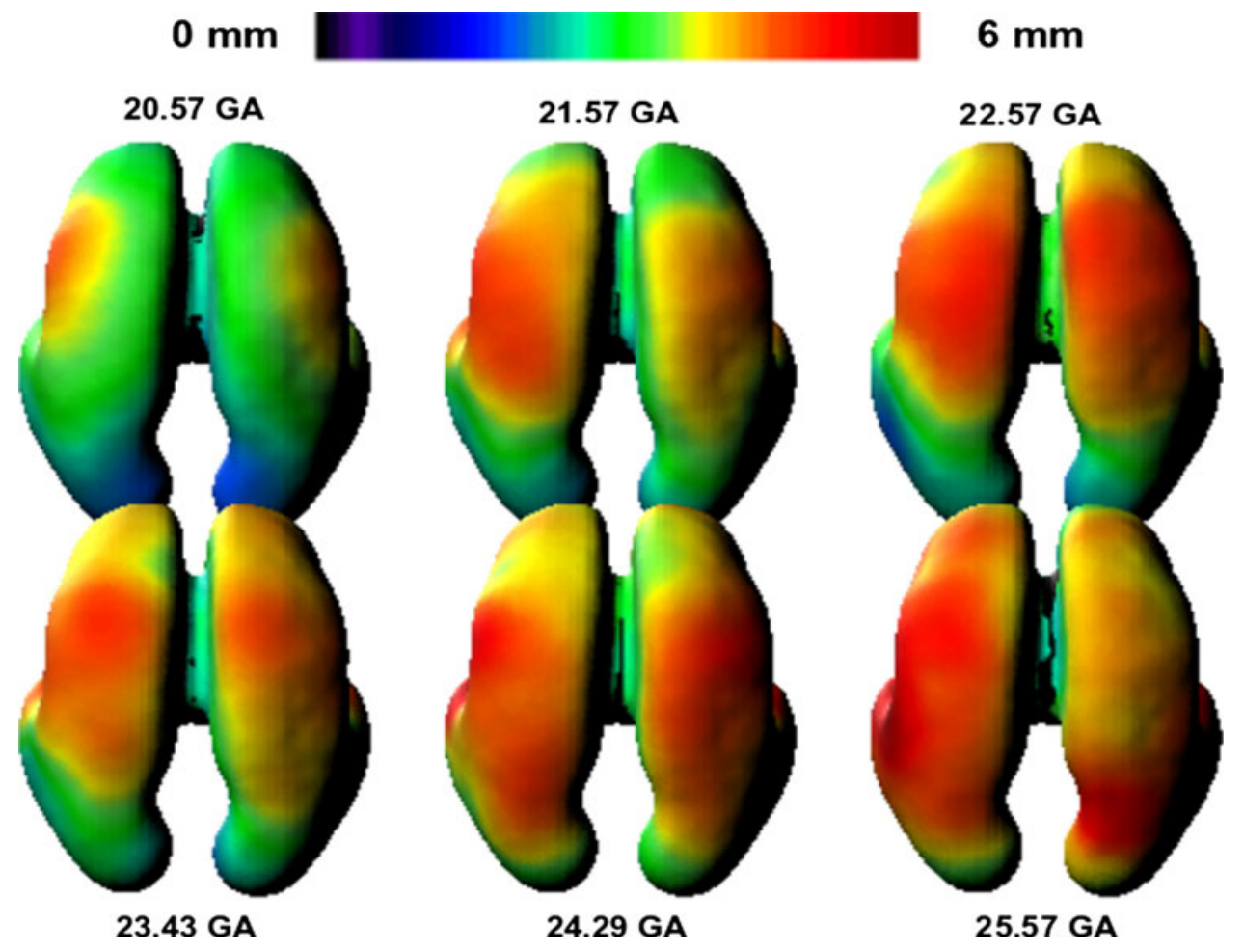

As previously stated, we used 3D information to compute the thickness of the SP and CP. Though the complexity of the CP and subplate shape from 20 to $26 \mathrm{GW}$ is much simpler than the convoluted cortex in the mature brain, this full 3D analysis still reduces error in estimating thickness in comparison to extracting 3D meaning from 2D images oriented with respect to a single plane of section (Jones et al. 2000). For our analysis, we computed the ratio of SP to CP thickness at each vertex along the average surface map. Previous studies report a maximum ratio of SP to CP thickness to be 5:1 (Kostovic and Rakic 1990; Kostovic et al. 2002; Perkins et al. 2008). However, we only found a maximum ratio value of just below 7:2 for an individual subject of $25.83 \mathrm{GW}$. One plausible factor for this discrepancy is that we estimate a true thickness measure between inner and outer surfaces of the SP, rather than simply taking an straight line distance between two fixed points on the inner and outer surfaces in 2D slices, which can yield different values (Jones et al. 2000). Secondly, our $\mathrm{CP}$ segmentation may be slightly overestimated, due to partial volume effects, or contrast differences in the clinical MRI compared to other studies, which could then have a negative effect on the thickness ratio. Despite these differences in values, the relative pattern of SP thickness and the SP to STV ratio are complementary to 2D studies (Perkins et al. 2008; Widjaja et al. 2010), but provide a more complete picture of the changes occurring over the brain. In general, greater SP to $\mathrm{CP}$ ratio values occurred in regions of greater SP thickness. This reflects the relatively 


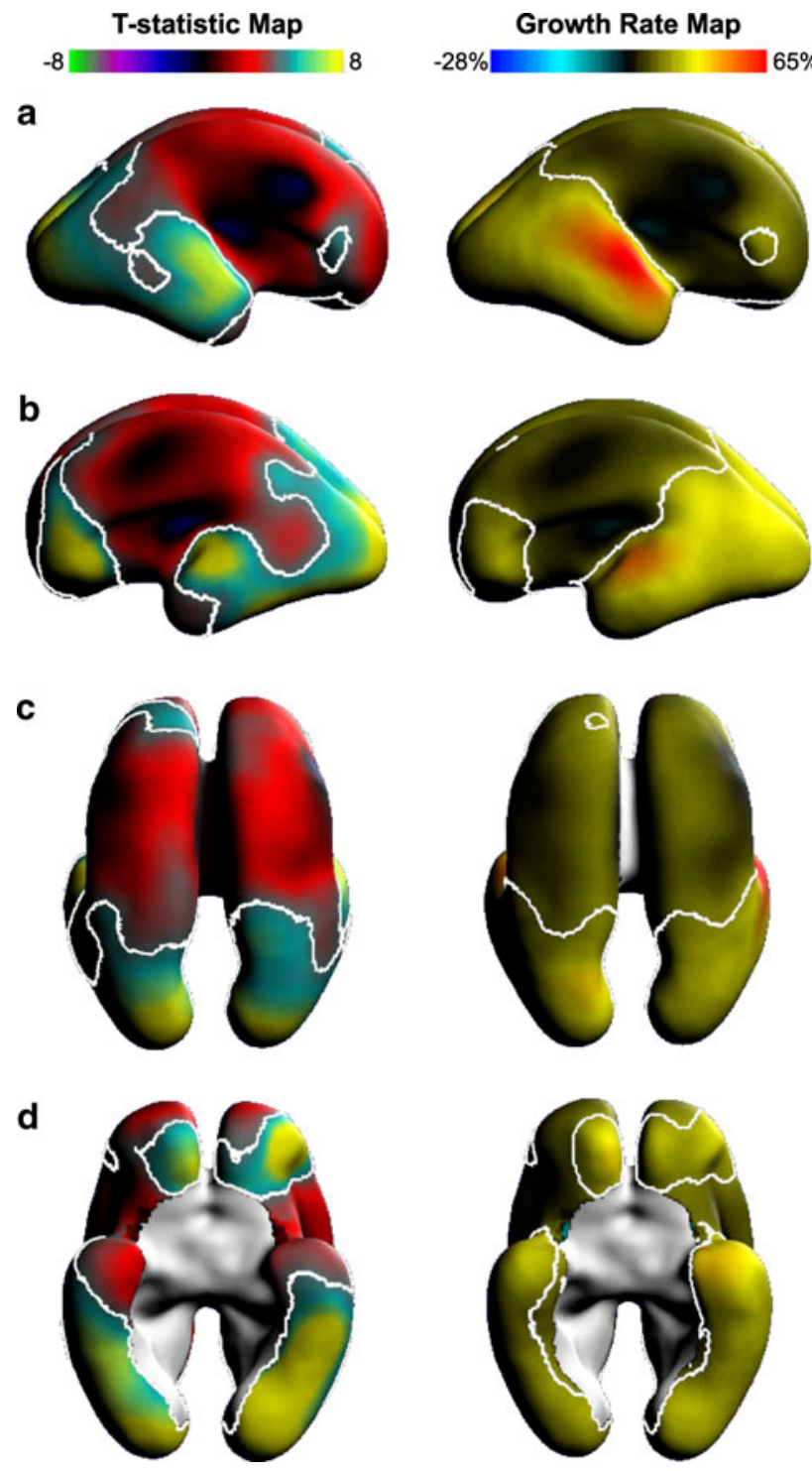

Fig. 7 Maps showing regional change in SP thickness and the corresponding growth rate (\% growth/GW) shown on the average fetal anatomy. In the first column, white contour outlines regions of significant change in SP thickness on a T-statistic map (corrected, $p<0.05$ ). In the second column, estimates for local percentage weekly thickness increases are shown for the study period. The full range of growth rates are displayed as colors together with an iso-rate contour indicating those points with $20 \%$ weekly growth rate. The (a) left and (b) right lateral hemispheres highlight the fastest growth rates along the superior and lateral aspects of the temporal lobe. The dorsal view (c) illustrates clear SP thickness increases (approximately $30 \% / \mathrm{GW}$ ) in the occipital poles. Nearly the entire ventral surface of the brain (d) shows significant positive change in SP thickness (approximately 30\%/GW)

uniform and slowly changing $\mathrm{CP}$ thickness, contrasting with the spatially varying and temporally thickening SP.

Though interesting developments in the SP continue into the third trimester of gestation, the age range of our study was limited by the converging T2-W intensities of SP and
IZ that occurs between 25 and $26 \mathrm{GW}(23 / 24 \mathrm{PCW})$ due changes in the tissue composition (Kostovic et al. 2002). The boundary between SP and IZ is visible on in utero MR T2-W images up to 27 GW (Prayer et al. 2006). However, we found that we could not reliably locate the deep boundary of the SP in all areas in order to calculate accurate volume and thickness. In the only other quantitative study of the SP in utero (Perkins et al. 2008), measurements of 2D thickness and diameters were collected up to $29 \mathrm{GW}$ on $\mathrm{T} 2-\mathrm{W}$ images, but only in selected regions. Post-mortem fetal MRI studies, which do not have the same time and parameter limitations for acquisition due to fetal motion and clinical requirements, find that contrast between the SP and IZ diminishes between 29 and $32 \mathrm{GW}$ (27-30 PCW) on T1-W images such that only the upper part of the SP is distinct (Kostovic et al. 2002) and after 25 GW (23 PCW) on T2-W images (Widjaja et al. 2010). The variability in the age at which contrast is lost is sensitive to the imaging parameters and the observer's subjective judgment. For our study, we calculated the mean intensity ratio between SP and IZ to demonstrate that these zones become iso-intense, on average, around $26 \mathrm{GW}$ on typical clinically acquired T2-W images and therefore segmentation must be limited to earlier gestational ages. The SP may be studied at later fetal and postnatal ages with inclusion of diffusion tensor imaging (DTI), which is not prone to the same iso-intensity challenges, but has other limitations (Trivedi et al. 2009; Widjaja et al. 2010). Specifically, infants born preterm are vulnerable to hypoxia-ischemia of which the subplate neurons are sensitive. MRI in conjunction with DTI may help to characterize the relationship between the $\mathrm{CP}$ with that of damaged subplate zone (McQuillen et al. 2003).

Alteration of the subplate may also be associated with abnormal development that can be detected on fetal MRI, such as ventriculomegaly or cases of agenesis of the corpus callosum, and our understanding of the structural consequences may be quantified by the techniques used here for normally developing brains. For example, without the corpus callosum, the lack of transverse cortico-cortical fibers may cause regional decreases in volume and thickness of the SP.

To date, this is the largest in utero study of SP development and the only 3D analysis of volume and thickness of the SP. We demonstrated that the SP can be segmented over its entire volume up to $26 \mathrm{GW}$. From this segmentation, we charted the spatio-temporal pattern of growth. We found that the SP exhibits regionally specific growth patterns during the waiting period that are associated with the development of cortical inputs. With further use of 3D motion correction, reconstruction and quantitative image analysis techniques like those used in this study, we are hopeful that our knowledge of early structural changes in 
the human fetal brain in utero can continue to be improved and expanded upon.

Acknowledgments This research was primarily supported by NIH/ NINDS Grant R01 NS055064, additional funding for imaging was from NIH/NINDS Grant K23 NS52506-03, and NIH/NCRR UCSFCTSI Grant No. UL1 RR024131. The authors are grateful to Addison Cuneo for her assistance.

Open Access This article is distributed under the terms of the Creative Commons Attribution Noncommercial License which permits any noncommercial use, distribution, and reproduction in any medium, provided the original author(s) and source are credited.

\section{References}

Bayer SA, Altman J (2005) The human brain during the second trimester (Atlas of the human central nervous system development). CRC Press, Indianapolis

Bystron I, Blakemore C, Rakic P (2008) Development of the human cerebral cortex: Boulder Committee revisited. Nat Rev Neurosci 9:110-122

Chan WY, Lorke DE, Tiu SC, Yew DT (2002) Proliferation and apoptosis in the developing human neocortex. Anat Rec 267:261-276

Girard NJ, Raybaud CA (1992) In vivo MRI of fetal brain cellular migration. J Comput Assist Tomogr 16:265-267

Glenn OA (2009) Normal development of the fetal brain by MRI. Semin Perinatol 33:208-219

Habas PA, Kim K, Corbett-Detig JM, Rousseau F, Glenn OA, Barkovich AJ, Studholme C (2010a) A spatiotemporal atlas of MR intensity, tissue probability and shape of the fetal brain with application to segmentation. Neuroimage 53:460-470

Habas PA, Kim K, Rousseau F, Glenn OA, Barkovich AJ, Studholme C (2010b) Atlas-based segmentation of developing tissues in the human brain with quantitative validation in young fetuses. Hum Brain Mapp 31:1348-1358

Hevner RF, Zecevic N (2006) Pioneer neurons and interneurons in the developing subplate: Molecular markers, cell birthdays, and neurotransmitters. In: Erzurumlu RS et al (eds) Development and plasticity in sensory thalamus and cortex 1st edn. Springer, New York, pp 1-17

Jones SE, Buchbinder BR, Aharon I (2000) Three-dimensional mapping of cortical thickness using Laplace's equation. Hum Brain Mapp 11:12-32

Kim K, Habas PA, Rousseau F, Glenn OA, Barkovich AJ, Studholme C (2010) Intersection based motion correction of multislice MRI for 3D in utero fetal brain image formation. IEEE Trans Med Imaging 29:146-158

Kostovic I, Goldman-Rakic PS (1983) Transient cholinesterase staining in mediodorsal nucleus of the thalamus and its connections in the developing human and monkey brain. J Comp Neurol 219:431-447

Kostovic I, Jovanov-Milosević N (2006) The development of cerebral connections during the first 20-45 weeks gestation. Semin Fetal Neonatal Med 11:415-422

Kostović I, Judas M (2010) The development of the subplate and thalamocortical connections in the human foetal brain. Acta Paediatr 99:1119-1127

Kostovic I, Rakic P (1990) Developmental history of the transient subplate zone in the visual and somatosensory cortex of the macaque monkey and human brain. J Comp Neurol 297:441-470

Kostovic I, Judas M, Rados M, Hrabac P (2002) Laminar organization of the human fetal cerebrum revealed by histochemical markers and magnetic resonance imaging. Cereb Cortex 12:536-544

Larroche JC, Feess-Higgins A (1987) Development of the human fetal brain: an anatomical atlas. INSERM, Paris

McQuillen PS, Ferriero DM (2005) Perinatal subplate neuron injury: implications for cortical development and plasticity. Brain Pathol 15:250-260

McQuillen PS, Sheldon RA, Shatz CJ, Ferriero DM (2003) Selective vulnerability of subplate neurons after early neonatal hypoxiaischemia. J Neurosci 23:3308-3315

Nichols TE, Holmes AP (2002) Nonparametric permutation tests for functional neuroimaging: a primer with examples. Hum Brain Mapp 15:1-25

Perkins L, Hughes E, Srinivasan L, Allsop J, Glover A, Kumar S, Fisk N, Rutherford M (2008) Exploring cortical subplate evolution using magnetic resonance imaging of the fetal brain. Dev Neurosci 30:211-220

Prayer D, Kasprian G, Krampl E, Ulm B, Witzani L, Prayer L, Brugger PC (2006) MRI of normal fetal brain development. Eur J Radiol 57:199-216

Rados M, Judas M, Kostović I (2006) In vitro MRI of brain development. Eur J Radiol 57:187-198

Rakic P (1976) Prenatal genesis of connections subserving ocular dominance in the rhesus monkey. Nature 261:467-471

Rousseau F, Glenn OA, Iordanova B, Rodriguez-Carranza C, Vigneron DB, Barkovich AJ, Studholme C (2006) Registration-based approach for reconstruction of high-resolution in utero fetal MR brain images. Acad Radiol 13:1072-1081

Samuelsen GB, Larsen KB, Bogdanovic N, Laursen H, Graem N, Larsen JF, Pakkenberg B (2003) The changing number of cells in the human fetal forebrain and its subdivisions: a stereological analysis. Cereb Cortex 13:115-122

Trivedi R, Husain N, Rathore RKS, Saksena S, Srivastava S, Malik GK, Das V, Pradhan M, Pandey CM, Gupta RK (2009) Correlation of diffusion tensor imaging with histology in the developing human frontal cerebrum. Dev Neurosci 31:487-496

Widjaja E, Geibprasert S, Mahmoodabadi SZ, Blaser S, Brown NE, Shannon P (2010) Alteration of human fetal subplate layer and intermediate zone during normal development on MR and diffusion tensor imaging. Am J Neuroradiol 31:1091-1099

Yezzi AJ Jr, Prince JL (2003) An Eulerian PDE approach for computing tissue thickness. IEEE Trans Med Imaging 22:1332-1339 